\title{
Attenuated Bacteria as Immunotherapeutic Tools for Cancer Treatment
}

\author{
Suneesh Kaimala ${ }^{1}$, Ashraf Al-Sbiei ${ }^{2}$, Otavio Cabral-Marques ${ }^{3}$, Maria J. Fernandez- \\ Cabezudo ${ }^{2}$ and Basel K. Al-Ramadi ${ }^{1 *}$
}

'Department of Medical Microbiology and Immunology, College of Medicine and Health Sciences, United Arab Emirates University, Al Ain, United Arab Emirates, ${ }^{2}$ Department of Biochemistry, College of Medicine and Health Sciences, United Arab Emirates University, Al Ain, United Arab Emirates, ${ }^{3}$ Center for Chronic Immunodeficiency (CCI), Medical CenterUniversity of Freiburg, Faculty of Medicine, University of Freiburg, Freiburg, Germany

\section{OPEN ACCESS}

Edited by:

Masoud H. Manjili, Virginia Commonwealth University, United States

Reviewed by: Michael Kogut,

Agricultural Research Service (USDA), United States

Caitlin S. L. Parello,

Biomodels, LLC, United States

*Correspondence: Basel K. Al-Ramadi ramadi.b@uaeu.ac.ae

Specialty section: This article was submitted to Cancer Immunity and Immunotherapy, a section of the journal Frontiers in Oncology

Received: 22 March 2018 Accepted: 16 April 2018

Published: 01 May 2018

Citation:

Kaimala S, Al-Sbiei A, CabralMarques O, FernandezCabezudo MJ and Al-Ramadi BK

(2018) Attenuated Bacteria as Immunotherapeutic Tools for Cancer

Treatment.

Front. Oncol. 8:136. doi: 10.3389/fonc.2018.00136
The use of attenuated bacteria as cancer therapeutic tools has garnered increasing scientific interest over the past 10 years. This is largely due to the development of bacterial strains that maintain good anti-tumor efficacy, but with reduced potential to cause toxicities to the host. Because of its ability to replicate in viable as well as necrotic tissue, cancer therapy using attenuated strains of facultative anaerobic bacteria, such as Salmonella, has several advantages over standard treatment modalities, including chemotherapy and radiotherapy. Despite some findings suggesting that it may operate through a direct cytotoxic effect against cancer cells, there is accumulating evidence demonstrating that bacterial therapy acts by modulating cells of the immune system to counter the growth of the tumor. Herein, we review the experimental evidence underlying the success of bacterial immunotherapy against cancer and highlight the cellular and molecular alterations in the peripheral immune system and within the tumor microenvironment that have been reported following different forms of bacterial therapy. Our improved understanding of these mechanisms should greatly aid in the translational application of bacterial therapy to cancer patients.

Keywords: bacterial therapy, attenuated Salmonella, cancer immunotherapy, tumor microenvironment, tumorinfiltrating leukocytes, myeloid-derived suppressor cells

The practice of using bacteria for cancer therapy dates back to the nineteenth century. In 1893, William Coley, a New York-based physician, prepared a filtered mixture of bacteria and bacterial lysates, composed of Streptococcus pyogenes and Bacillus prodigiosus (now called Serratia marcescens) and called it "Coley's Toxin." He found that, in some cases, the tumors regress when Coley's toxin is injected into the tumors (1). Later on he developed a safe vaccine, a mixture of heat killed S. pyrogenes and Seretia marcescenes, to successfully treat sarcoma, carcinoma, lymphoma, melanoma, and myeloma (2). These procedures practiced by Coley formed the basis of the recent advances in the cancer immunotherapy using attenuated bacterial strains. Today, the most common species of bacteria being used as immunotherapeutic agents are Clostridium novyi $(3,4)$, Listeria monocytogenes $(5,6)$, and Salmonella enterica serovar Typhimurium (hereafter referred to as Salmonella typhimurium) (6-10). Infection of poorly antigenic tumors with facultative anaerobic bacteria is thought to increase their antigenicity. Bacterial infections also alter the function of different cellular components of the immune system, such as $\mathrm{CD}^{+}$and $\mathrm{CD} 8^{+} \mathrm{T}$ cells, myeloid-derived suppressor cells (MDSCs), regulatory T cells (Tregs), tumor-associated macrophages (TAMs), and their activation. Many conserved bacterial ligands are agonists for innate immune system receptors, such as 
toll-like receptors (TLR), and upon binding, initiate an intracellular signaling cascade leading to the production of proinflammatory cytokines. Moreover, there is evidence that some bacterial components, such as exotoxins, may initiate anti-tumor activities not only by indirect activation of the immune system, but also by their direct action on tumor cells $(11,12)$. In addition to their immunotherapeutic properties against diverse types of cancers, Salmonella and Listeria are also used as vectors for delivering immunogenic tumor antigens to the host. The use of bacteria for delivering tumor antigens has been reviewed in detail elsewhere $(13,14)$. Here, we aim to review the direct effects of bacterial immunotherapeutic agents on different cellular components of the immune system.

\section{EFFECTS OF BACTERIAL THERAPY ON MYELOID CELLS}

\section{Tumor-Associated Macrophages}

As the tumor starts growing it gets enriched with different myeloid cell populations, such as MDSCs, tumor-associated neutrophils (TANs), TAMs, Tek tyrosine kinase receptor (TIE)2 -expressing monocytes, and tolerogenic dendritic cells $(15,16)$. Monocytes are recruited to sites of tumor growth in response to chemoattractants released by tumor cells, such as colony stimulating factor 1 and the chemokine C-C motif chemokine ligand 2 (CCL2) (17), where they differentiate into macrophages. Usually, these are alternatively activated cells involved in tissue repair, also known as M2-type macrophages (18), and they express immunosuppressive molecules, such as arginase 1 (Arg1) (19) and the cytokine IL-10 (20). M2 macrophages support the growth and malignancy of tumors by suppressing the host's antitumor immune responses. In contrast, M1 macrophages, also known as classically activated or killer macrophages, express nitric oxide synthase $\left(\mathrm{NOS}_{2}\right)$ and TNF- $\alpha$ and orchestrate protective anti-tumor immune responses $(21,22)$.

We have recently reported that treatment of mice bearing B16.F1 melanoma with an attenuated strain of S. typhimurium led to maturation of intratumoral myeloid cells and diminished their suppressive capacity, enhancing the host's anti-tumor immune responses, and eventually leading to tumor regression (23). Multi-color flow cytometric analysis of tumor-infiltrating leukocytes (TILs) revealed that Salmonella induced tumor inhibition (Figure 1A) results in increased immune responses in the tumor microenvironment. This fact is evidenced by the significantly elevated levels of TILs in the tumors of Salmonella-

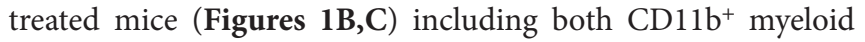
cells (Figures 1D,E) and CD $4^{+}$and $\mathrm{CD} 8^{+} \mathrm{T}$ cells (Figures 1F-I). Analysis of the different subpopulations of tumor-associated myeloid cells showed that Salmonella treatment led to a large increase in the proportion of cells characterized by being $\mathrm{CD}_{11 \mathrm{~b}} / \mathrm{F} 4 / 80^{+} / \mathrm{Ly} 6 \mathrm{C}^{-} / \mathrm{Ly} 6 \mathrm{G}^{-}$, which are commonly known as TAMs (Figure 1J). Upon treatment with Salmonella, TAMs increase the expression of M1-type macrophage activation markers, such as the IFN- $\boldsymbol{\gamma}$-dependent Sca- 1 and MHC class II proteins (Figures $\mathbf{1 K}-\mathbf{N}$ ). Representative FACS plots of the data highlighting the gating strategy for untreated (Figure 1T) and
Salmonella-treated tumors (Figure 1U) are also shown. This observation indicates that treatment with Salmonella skews the TAMs profile, reprogramming them toward proinflammatory functions and indicating the functional plasticity of M1/M2 macrophages. A similar shift in the phenotypic characteristics of macrophages was reported by Hong and coworkers upon intratumoral injection of a recombinant, attenuated S. typhimurium vaccine into Her2/Neu-expressing CT26 tumors (24). Following the injections, the phenotype of the splenic and intratumoral macrophage populations was shifted from an immature to mature-type expressing TNF $\alpha$ (24). Treatment of mice bearing an aggressive ID8-Defb29/Vegf-A ovarian carcinoma with an attenuated strain of $L$. monocytogenes $(\Delta \mathrm{actA} / \Delta \mathrm{inlB})$ also resulted in augmented infiltration of macrophages into the tumor and in a shift from their M2 to M1 profile (25). These macrophages displayed elevated phagocytic and tumoricidal activity. Moreover, TAMs in the peritoneal exudates of treated mice exhibited increased expression of the co-stimulatory molecules CD80 and CD86, increased gene expression of proinflammatory cytokines, and downregulated transcriptional activity of suppressive effector molecules (25).

\section{Dendritic Cells and TANs}

Of note, it was recently reported that murine or human melanoma cell lines infected with pathogenic L. monocytogenes $\left(\mathrm{LM}^{\mathrm{WT}}\right)$, transformed these malignant melanocytes into professional antigen-presenting cells (APCs) with a phenotype and function analogous to those of skin DCs (5). These infected melanoma cells expressed phenotypic markers, such as CD11c, F4/80, MHCII, CD40, and CD83 similar to mature dendritic cells (5). The mechanisms explaining these phenotypic changes by invasive pathogens, such as Salmonella, consist in their capacity to stimulate TLR signaling pathways, which also enhance the expression of co-stimulatory molecules (e.g., CD86 and CD80) on APCs (26). Such events, enable APCs to strongly activate antigen-specific CD8 T cells and natural killer (NK) cells, which in turn, mediate tumor killing and regression. Most TLRs signal through myeloid differentiation primary response 88 (MyD88), an essential cytoplasmic adaptor protein that links triggering of TLRs and IL-1/IL-18 receptors with downstream activation of IL-1 receptor-associated kinases (IRAKs) and NF- $\mathrm{KB}$ (27). Treatment of MyD88-deficient mice carrying B16.F1 tumors with attenuated Salmonella was ineffective in regressing tumor growth, indicating that bacterial therapy of tumors is dependent on TLR-MyD88 signaling (23). These findings suggest that the anti-tumor effect of attenuated Salmonella is mediated through changes in intratumoral myeloid cells.

The effect of Salmonella immunotherapy on TANs has also been reported. Treatment of the mammary LM3 adenocarcinoma with a Salmonella typhi vaccine strain led to the activation and recruitment of neutrophils into the tumor site. These neutrophils were able to secrete TNF $\alpha$, which in combination with IFN $\gamma$, exerted synergistic cytotoxic effects on endothelial and tumor cells within the tumor microenvironment (28). Recruitment of TANs into the tumor tissue was observed when a B-cell nonHodgkin lymphoma was intratumorally injected with the attenuated S. typhimurium strain LVR01. This treatment elicited both 


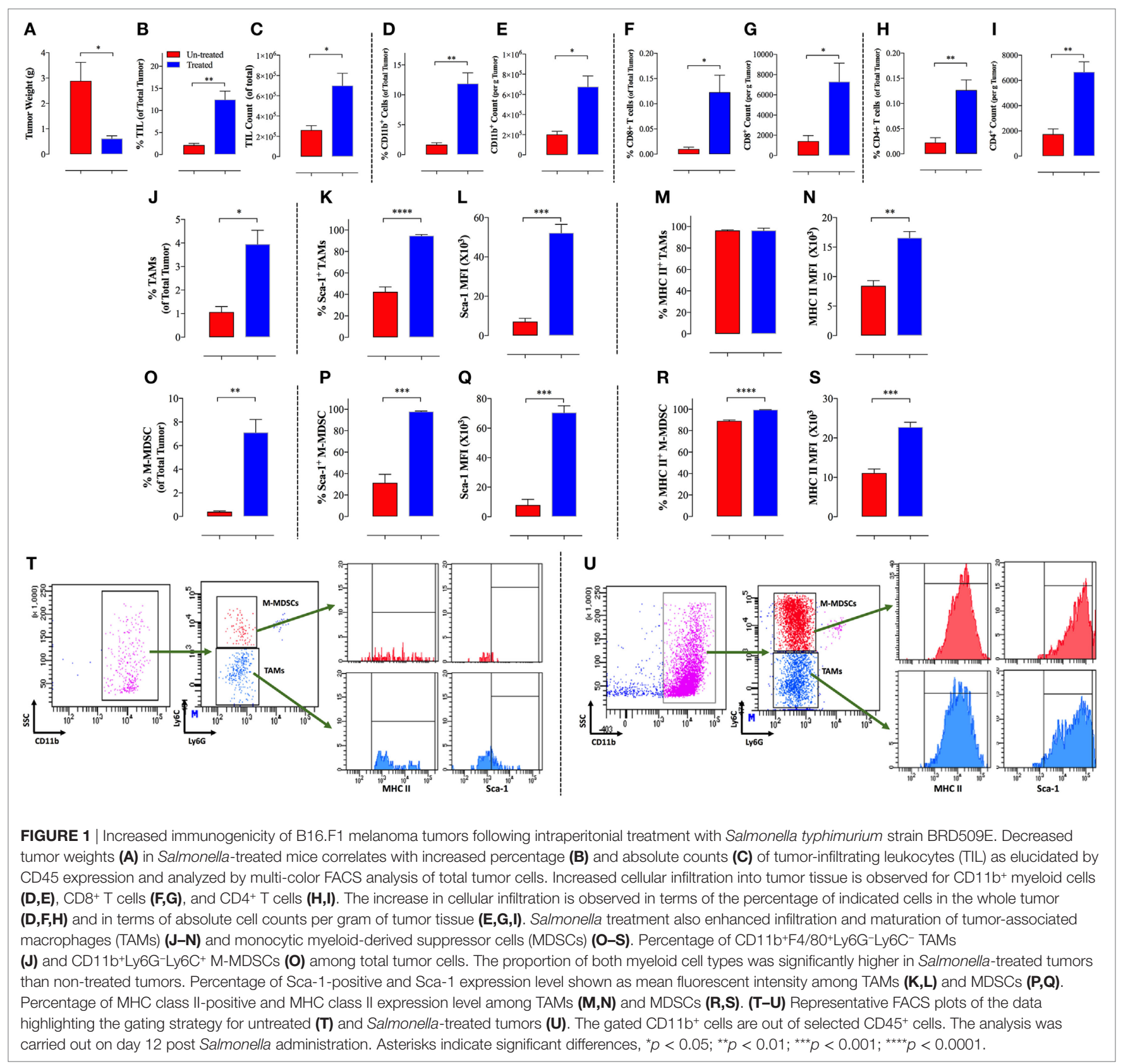

local and systemic anti-tumoral immune responses, eventually leading to enhanced host survival (29). The exact cause of the phenotypic changes in myeloid cells during bacterial immunotherapy is not known. Such alterations in intratumoral myeloid cells have also been reported during cancer therapy using the parasitic protozoan Toxoplasma gondii $(30,31)$. It is likely that stimulation of TLR signaling by various bacterial components could account for these effects (32).

\section{EFFECTS OF BACTERIAL THERAPY ON TUMOR-ASSOCIATED LYMPHOID CELLS}

The tumor microenvironment harbors lymphoid subpopulations, such as NK cells, B cells, CD4 ${ }^{+} \mathrm{T}$ cells, $\mathrm{CD} 8^{+} \mathrm{T}$ cells, and Tregs
$(15,16)$. The lymphoid cells can be tumor promotive or suppressive in nature. The tumor microenvironment is a critical factor that influences the function of these lymphoid subpopulations in the tumor (33).

\section{NK Cells}

Natural killer cells are prototypical innate lymphoid cells able to recognize and eradicate tumor cells without prior antigenic exposure. However, immunosubversion (i.e., when the tumor suppresses the host immune system) as well as immunoediting or immunoselection (i.e., outgrowth of poorly immunogenic tumor-cell variants) impairs NK cell responses to tumors $(34,35)$. We have previously shown that NK cells are readily activated by a recombinant Salmonella strain engineered to express IL-2 (36). 
Moreover, this recombinant strain exhibited superior anti-tumor activity against B16.F1 melanoma in a syngeneic tumor model, an activity that correlated with its capacity to induce a higher level of tumor cell killing in vivo (37). In addition, Salmonella strains expressing IFN- $\gamma$ or TNF- $\alpha$ have been shown to induce highly effective immune-potentiating anti-microbial as well as anti-tumoral responses (38-41). The enhanced anti-tumoral capacity of an engineered IFN- $\gamma$-expressing Salmonella strain was dependent on the activation of NK cells (41). A recent study reported that treatment of non-Hodgkin lymphoma-bearing mice with attenuated Salmonella resulted in increased expression of chemokines, such as $\mathrm{Ccl} 2, \mathrm{Ccl} 3$, and $\mathrm{Ccl} 5$, which are involved in the recruitment of NK cells, and enhanced NK cell cytotoxic activity against target cells (42).

\section{$\mathrm{CD4}^{+}$and $\mathrm{CD}^{+}{ }^{+} \mathrm{T}$ Cells}

The anti-tumor functions of CD4 T-cell depend upon their specific subset (Th1, Th2, or Th17 cells) (43). Th1 cells are antitumorigenic by virtue of either activating CD8 T cells (44) or directly killing tumor cells by secreting TNF- $\alpha$ and/or IFN- $\gamma$ (45). Th1 cells also act against tumor development by licensing DCs and macrophages, e.g., increasing their antigen-presenting potential that enable $\mathrm{T} \mathrm{CD} 8^{+}$cells to develop a strong cytotoxic activity (45). On the other hand, while Th2 cells are less relevant to cancer pathology, Th17 cells found within the tumor microenvironment facilitate tumor growth by the secretion of IL-17, a pro-angiogenic cytokine (46).

In the B16.F1 melanoma model, intraperitoneal treatment with attenuated Salmonella led to increased infiltration of both $\mathrm{CD}^{+}$and $\mathrm{CD}^{+} \mathrm{T}$ cells into the tumor (Figures 1F-I). Both $\mathrm{T}$ cell types were increased by $>$ threefold in terms of percentage within total tumor cells and in their absolute counts per weight of tumor tissue. CCL5 and macrophage inflammatory protein 1 alpha, which are secreted by proinflammatory M1 macrophages, are known to recruit activated T cells into the tumor (47). Hence, this represents a mechanism by which activated macrophages induced by Salmonella treatment could be responsible for the enhanced $\mathrm{T}$ cell infiltration into tumors. Several studies have reported similar effects as a result of administration of attenuated bacteria in different models. When mice bearing EL4 lymphoma were immunized with Salmonella typhi by injecting the bacteria into the tumor and the draining lymph node areas, the leukocyte populations in the tumor draining lymph nodes were expanded and tumor growth was significantly decreased (48). The tumors in treated mice contained significantly decreased levels of IL-10 and this was accompanied by a reduction in the mitotic index of tumors, a delayed development of palpable lymph node metastases and, most importantly, increased survival compared to untreated mice. In another study, an enhancement of tumor-infiltrating activated CD8 T cells was observed following treatment of mice carrying A20 lymphoma with the attenuated S. typhimurium LVR-01 strain, which consequently led to a reduction in tumor growth (29). When the vaccine was supplemented with IL-2, there was an increased infiltration of CD4 and CD8 T cells in the tumors. The combined treatment resulted in better control of tumor growth and improved animal survival significantly over the Salmonella vaccine alone treatment (49). This effect is similar to our previously reported findings demonstrating the superior anti-tumor activity of an IL2-expressing Salmonella strain in the melanoma model (37). Thus, treatment of the tumors with Salmonella alone or in combination with interleukins potentiates the tumoricidal activity of tumor-infiltrating lymphocytes. Moreover, there is direct evidence that cytokine-expressing Salmonella strains can strongly modulate macrophage activation and function (40). Using CD4 and CD8 T cell-deficient mice, Lee and coworkers showed that Salmonella treatment of Lewis lung carcinoma (LL2)-bearing mice was relatively less efficient in the absence of T cells (34-42\% inhibition in tumor growth compared to $50 \%$ in WT mice) (50). Systemic treatment with Salmonella induced a Th1 inflammatory response at the tumor site, which was accompanied by macrophage and neutrophil infiltration and a significant increase in IFN $\gamma$ production (50). Similarly, immunization of mice bearing a subcutaneous mammary tumor (LM3 adenocarcinoma) with attenuated $S$. typhi in the peritumoral tissue and the tumor draining lymph nodes initiated an anti-tumor Th1 response that was characterized by increased frequencies of IFN $\gamma$-secreting $\mathrm{CD}^{+}$and $\mathrm{CD} 8^{+} \mathrm{T}$ cells within the tumor. Treated mice displayed a reduced tumor growth and lung metastasis, and prolonged survival compared to unimmunized mice (28). In another study, when mice bearing Her2/neu-expressing CT-26 tumors were injected with a recombinant attenuated S. typhimurium vaccine intratumorally, a significant reduction in tumor growth was evidenced. This effect on tumor growth was partially lost upon depletion of CD8 T cells, suggesting a role for these cells in Salmonella-mediated tumor suppression (24). Using the attenuated S. typhimurium strain A1-R, which was originally derived by in vivo passaging through tumor tissue (51), Murakami and colleagues recently demonstrated its efficacy in promoting CD8 $\mathrm{T}$ cell infiltration and tumor growth arrest in a syngeneic pancreatic-cancer orthotopic mouse model (52). The immunotherapeutic effect of L. monocytogenes is also partially mediated by cytotoxic $\mathrm{T}$ lymphocytes (CTLs). Immunization of mice carrying triple negative mammary tumors (4T1 cells) with Listeria led to direct killing of tumor cells by the bacteria, eventually leading to the eradication of the primary tumor and all the metastases. Depletion of CD8 T cells partially restored tumor growth in these mice, indicating that CTLs also play an important role in Listeria-mediated tumor therapy (53). Finally, treatment of ovarian carcinoma with attenuated strain of the parasite T. gondii (CPS) led to an increase in $\mathrm{CD}^{+}$and $\mathrm{CD}^{+}$ $\mathrm{T}$ cell infiltration into the tumor microenvironment, activation of tumor-resident $\mathrm{T}$ cells, and enhanced IFN- $\gamma$ production by $\mathrm{T}$ cell populations (31). Taken together, these data highlight the immune-potentiating, anti-tumor effects of bacterial therapy in diverse cancer models.

Bacterial immunotherapeutic agents can induce memory $\mathrm{T}$ cell responses as well. Treatment of mice harboring established hepatic metastases of colorectal cancers with an attenuated strain of L. monocytogenes expressing a tumor-associated antigen led to a strong initial tumor specific CD8 T cell response that successfully treated $90 \%$ of the animals (54). It also generated central and effector memory $\mathrm{T}$ cells that protected the mice against tumor re-challenge. Additionally, the treatment led to a decrease in the expression of PD-1, an immune inhibitory molecule expressed 
on tumor-infiltrating lymphocytes, demonstrating the efficacy of this vaccine to down modulate the immunosuppressive tumor microenvironment (54).

\section{Gamma-Delta $(\gamma \delta)$ T Cells}

Gamma-delta T cells are a subset of T lymphocytes characterized by the presence of a surface antigen recognition complex type 2 . Activated $\gamma \delta$ T cells exhibit potent anti-tumor activity by releasing copious amounts of IFN- $\gamma$ and TNF- $\alpha$ (55). Mycobacterial immunotherapy using Mycobacterium vaccae, $M$. obuense, or BCG induced the proliferation and activation of $\gamma \delta \mathrm{T}$ cells (56). Activated $\gamma \delta \mathrm{T}$ cells showed enhanced effector responses including upregulated granzyme expression and production of Th1 cytokines, such as IFN- $\gamma$ and TNF- $\alpha$ (56). When Listeria moncytogenes was used as immunotherapeutic agent against cervical cancer in mice, increased levels of intratumoral IL-17 and IL-17-positive $\gamma \delta \mathrm{T}$ cells were observed (57). However, despite the activation of $\gamma \delta$ T cells by Listeria treatment in tumor-bearing mice, tumor progression was unaltered in $\gamma \delta \mathrm{T}$ cell-deficient mice. Instead, Listeria-induced anti-tumor activity was critically dependent on $\alpha \beta$ T cells. This indicated that, at least in this cervical cancer model, Listeria immunotherapy-associated $\gamma \delta \mathrm{T}$ cell activation is of secondary importance (57).

\section{EFFECT OF BACTERIAL THERAPY ON IMMUNOSUPPRESSIVE CELLS}

\section{Myeloid-Derived Suppressor Cells}

Myeloid-derived suppressor cells, the immature myeloid cells present in the bone marrow, peripheral blood, and spleen under normal physiological conditions, can undergo tremendous expansion in various pathological conditions, such as cancers and autoimmune diseases (58). Recruitment of MDSCs and Tregs into the tumor microenvironment helps tumors to evade the host immunosurveillance system. In cancers, these cells suppress CD 4 and CD8 T cell-mediated anti-tumor responses by the nitration of the T cell receptors (59). Hence, MDSCs are regarded as one of the most important targets for cancer therapy. Several drugs that differentiate MDSCs into mature phenotypes are in use for cancer therapeutic purposes.

Recently, we demonstrated that treatment of B16.F1 melanoma with an attenuated strain of $S$. typhimurium leads to an

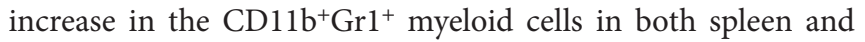
tumor (23). Subsequent to Salmonella treatment, intratumoral myeloid cells exhibited a significant loss in their immunosuppressive capacity. Interestingly, this phenotype shift was not observed among the splenic myeloid cells, suggesting that the splenic and intratumoral myeloid cells respond differentially to Salmonella treatment. Moreover, we observed an increase in the expression of co-stimulatory molecules, such as CD40, CD80, and CD86 on myeloid cells in the treated group of mice, suggesting that the myeloid cells shifted from an immature to mature phenotype following Salmonella inoculation (23). Further analysis of intratumoral MDSCs indicated that Salmonella treatment resulted in a significant increase in the proportion of M-MDSCs (Figure 10). Furthermore, these cells increased their expression of the differentiation markers Sca-1 (Figures 1P,Q) and MHC class II (Figures 1R,S), indicative of their activity level and maturation status. This suggested that Salmonella treatment triggered the differentiation of intratumoral MDSCs, causing a reduction in their immunosuppressive properties.

Wallecha and coworkers demonstrated that cancer immunotherapy using L. monocytogenes-LLO (Lm-LLO) diminished the suppressive functions of MDSCs and Tregs in the tumor microenvironment (60). This diminished suppressive function was linked to a reduction in the expression of Arg1 by MDSCs and IL-10 by Tregs (60). While the former decreases the expression of the T-cell receptor CD3 $\varsigma$ chain (19), the latter is a pleiotropic immunosuppressive cytokine (61). When used as an immunotherapeutic vaccine to treat 4T1 tumors in young and old aged mice, L. monocytogenes-infected MDSCs in the blood and the primary tumors causing a reduction in their number and converting them into immune-stimulating, IL12-producing macrophages. This was accompanied by a dramatic reduction in tumor metastasis and tumor growth (62). A recent study by Zhang and colleagues demonstrated the capacity of bacterial lipoprotein (BLP), a TLR1/2 agonist, to decrease intratumoral MDSC accumulation while allowing for infiltration by IFN $\gamma$-expressing $\mathrm{CD}^{+} \mathrm{T}$ cells (63). Using a syngeneic glioma mouse model, the authors showed that mice treated with a systemic injection of BLP and adoptively transferred antigen-specific $\mathrm{T}$ cells had improved survival. The alterations in MDSC and $\mathrm{CD}^{+} \mathrm{T}$ cells ratios within the tumor microenvironment were associated with increased expression of CXCL10, a chemokine that induces $\mathrm{CD}^{+} \mathrm{T}$ cell migration, and a reduction in the expression of CCL2, known to regulate the migration of MDSC (63).

\section{Regulatory T Cells}

Regulatory $\mathrm{T}$ cells are implicated in autoimmune diseases and cancers. Under healthy conditions, $\mathrm{CD} 4^{+} \mathrm{CD} 25^{+} \mathrm{Foxp}^{+}{ }^{+}$Tregs function to prevent immune reactions to autoantigens. However, these cells are known to attenuate anti-tumor immunity by inhibiting tumor antigen-specific CTLs (64). Recent studies showed that Tregs could be targets of bacterial immunotherapy against cancer. Peritumoral injection of attenuated $S$. typhi in mammary adenocarcinoma-bearing mice led to a reduction in $\mathrm{T}$ regulatory cells in tumor draining lymph nodes and led to decreased metastasis and enhanced overall host survival (28). Likewise, intratumoral injection of attenuated Salmonella reduced the percentage of $\mathrm{CD} 25^{+} \mathrm{FoxP}^{+}$cells among spleen and tumor $\mathrm{CD}^{+}$ $\mathrm{T}$ cells in a colon cancer model (24). The precise reason for the effect of Salmonella vaccines on Tregs is obscure. However, it is known that Salmonella treatment leads to the downregulation of CD44, a key cell surface molecule on Tregs as well as tumor cells, and contributes to tumor angiogenic invasiveness and proliferative potential (65). Interestingly, systemic administration of a synthetic BLP, a TLR1/TLR2 agonist, to mice with established lung carcinoma, melanoma, or leukemia, led to tumor regression and a long-lasting protective response against tumor re-challenge (66). The BLP-mediated immunotherapeutic effect was due to a reduction in the suppressive function of Tregs and a concomitant enhancement in anti-tumor CTL response (66). In another study, intraperitoneal administration of a $l p p A B / m s b B$ mutant of 


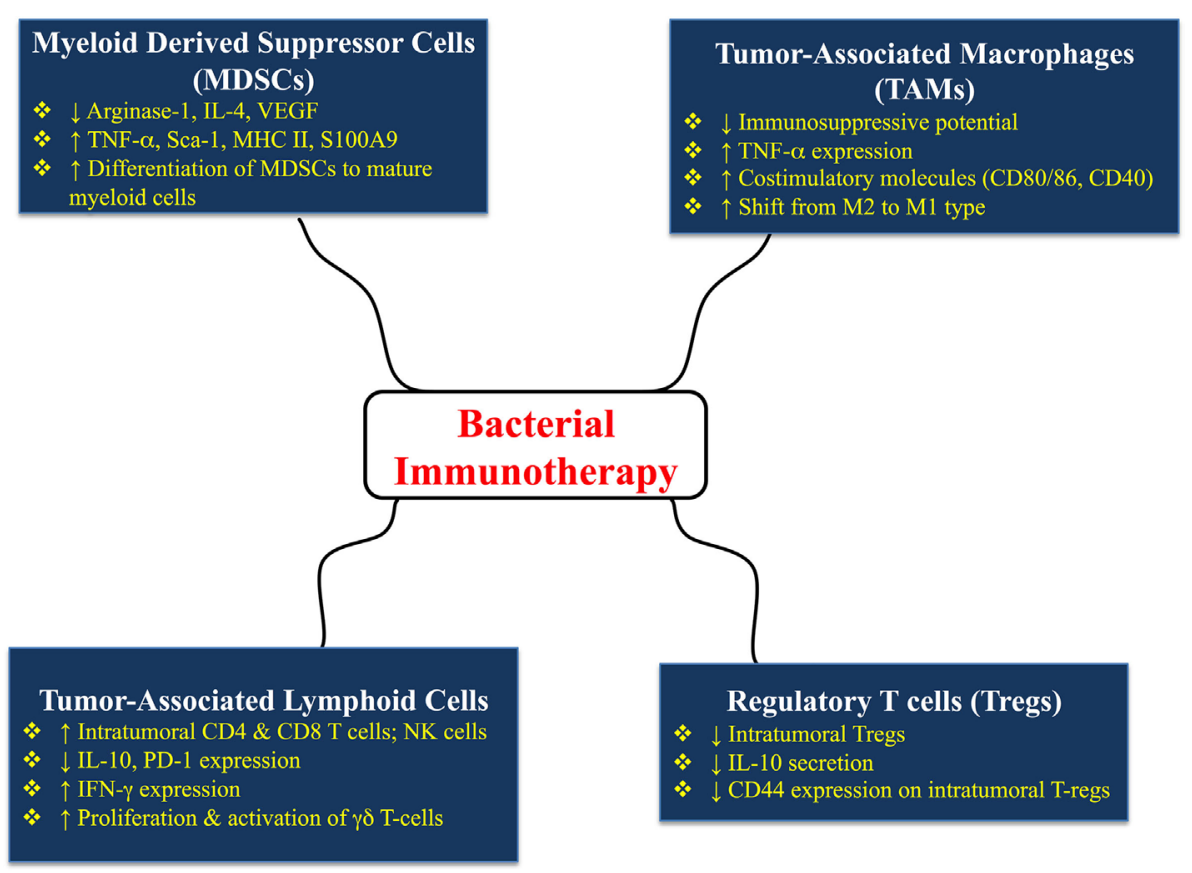

FIGURE 2 | Multiple effects of bacterial immunotherapy on different immune cell types in tumor-bearing hosts. The details and references for the summarized changes are discussed in the text.

S. typhimurium (lacking Braun lipoprotein; LPP) failed to induce anti-tumor activity against subcutaneously implanted B16.F10 tumors. This indicated that LPP is a critical factor for the antitumoral activity of attenuated Salmonella (65).

A schematic summary of the multiple effects of bacterial immunotherapy on different immune cell types is shown in Figure 2.

\section{CONCLUDING REMARKS}

More than 120 years after Coley's documentation of the effect of bacterial infections on human tumors, various forms of "Coley's toxins" are currently being actively developed as therapeutic agents to treat cancers (67). The greatest challenge for a wide acceptance of this approach has been the concerns about the safety of using potentially pathogenic bacteria for therapeutic purposes. In order to overcome this limitation, genetically modified organisms have been developed in such a way to balance the requirement for safety while maintaining therapeutic efficacy. There are many examples where engineered strains of Salmonella enterica serovar Typhimurium, L. monocytogenes, and Clostridium novyi-NT have been used in preclinical models as well as in clinical trials $(4,68-72)$. Although so far limited, a few examples of the use of attenuated bacteria in clinical trials have been reported. Intratumoral injection of C. novyiNT spores has been successfully used to treat a patient with advanced leiomyosarcoma (4). Moreover, the safety of attenuated $L$. monocytogenes strains has been demonstrated in patients with advanced cancers $(71,72)$.
Accumulating evidence has demonstrated that heavy alterations in the pathogenicity of bacteria through the introduction of mutations to alleviate safety concerns actually compromise their therapeutic potential. However, important achievements have improved this perspective. Notably, it was recently shown that mutations in genes involved in the shikimate pathway, such as aroA, attenuates Salmonella while enhancing their immunogenicity (73). Furthermore, mutations that lead to modifications in lipid A and flagella synthesis result in an increased immunestimulatory capacity and as such the mutant strain was able to overcome the efficacy-limiting effects of pre-exposure (74). Conditional expression of LPS by using an inducible promoter has been useful in enhancing the intrinsic anti-tumor effects of attenuated Salmonella strains (75).

It is currently clear that using live bacteria to treat cancer is a form of immunotherapy. However, the precise mechanisms underlying this process remain incompletely understood. The findings to date point to strong immunomodulatory effects of bacterial therapy within the tumor microenvironment. This is perhaps best illustrated in the case of Salmonella therapy where the accumulation of bacteria within the tumor tissue leads to increased immunogenicity of tumors. Given that myeloid cells are the natural habitat for Salmonella organisms, these cells appear to be the major modulatory targets of this form of anti-tumor therapy. Salmonella-mediated inhibition of B16.F1 melanoma was not compromised when tested in nude mice, suggesting that the anti-tumor effect of Salmonella therapy is thymusindependent (23). Instead, effective Salmonella therapy was totally abrogated in mice deficient in the TLR-MyD88 signaling 
pathway (23), implicating innate immune cells as the primary target of this intervention. It is likely that the contribution of the different arms of the immune system to Salmonella-mediated cancer control is highly dependent on the relative tumorigenicity and immunogenicity of the tumor being investigated. As demonstrated by findings in our laboratory and others, successful inhibition of tumor growth by Salmonella is associated with the transformation of suppressive M2-like myeloid cells to inflammatory M1-type mature macrophages, which in turn likely leads to an enhancement in anti-tumor T cell responses. The capacity of Salmonella organisms to induce changes in macrophage functions in infections and cancer is well established $(37,76,77)$. The effectiveness of anti-cancer bacterial therapy through targeting myeloid cells has refocused attention on the importance of these lymphoreticular "white" cells in tumors, first described by Virchow more than 150 years ago (78). Our increased understanding of the properties of bacteria that enhance their anti-tumor and immunostimulatory capacity, and their precise cellular and molecular targets within the tumor microenvironment, promises to usher in new modalities for cancer treatment.

\section{REFERENCES}

1. Coley WB. Disappearance of a recurrent carcinoma after injections of mixed toxins. Ann Surg (1912) 55:897-8.

2. Richardson MA, Ramirez T, Russell NC, Moye LA. Coley toxins immunotherapy: a retrospective review. Altern Ther Health Med (1999) 5(3):42-7.

3. Lambin P, Theys J, Landuyt W, Rijken P, van der Kogel A, van der Schueren E, et al. Colonisation of Clostridium in the body is restricted to hypoxic and necrotic areas of tumours. Anaerobe (1998) 4(4):183-8. doi:10.1006/ anae.1998.0161

4. Roberts NJ, Zhang L, Janku F, Collins A, Bai RY, Staedtke V, et al. Intratumoral injection of Clostridium novyi-NT spores induces antitumor responses. Sci Transl Med (2014) 6(249):249ra111. doi:10.1126/scitranslmed.3008982

5. Bronchalo-Vicente L, Rodriguez-Del Rio E, Freire J, Calderon-Gonzalez R, Frande-Cabanes E, Gomez-Roman JJ, et al. A novel therapy for melanoma developed in mice: transformation of melanoma into dendritic cells with Listeria monocytogenes. PLoS One (2015) 10(3):e0117923. doi:10.1371/ journal.pone.0117923

6. Shahabi V, Maciag PC, Rivera S, Wallecha A. Live, attenuated strains of Listeria and Salmonella as vaccine vectors in cancer treatment. Bioeng Bugs (2010) 1(4):235-43. doi:10.4161/bbug.1.4.11243

7. Wall DM, Srikanth CV, McCormick BA. Targeting tumors with Salmonella typhimurium-potential for therapy. Oncotarget (2010) 1(8):721-8. doi:10.18632/oncotarget.101208

8. Al-Ramadi BK, Fernandez-Cabezudo MJ, El-Hasasna H, Al-Salam S, Attoub S, $\mathrm{Xu} \mathrm{D}$, et al. Attenuated bacteria as effectors in cancer immunotherapy. Ann N Y Acad Sci (2008) 1138:351-7. doi:10.1196/annals.1414.036

9. Hoffman RM. Tumor-targeting Salmonella typhimurium A1-R: an overview. Methods Mol Biol (2016) 1409:1-8. doi:10.1007/978-1-4939-3515-4_1

10. Felgner S, Pawar V, Kocijancic D, Erhardt M, Weiss S. Tumour-targeting bacteria-based cancer therapies for increased specificity and improved outcome. Microb Biotechnol (2017) 10(5):1074-8. doi:10.1111/1751-7915.12787

11. Zhao M, Yang M, Li XM, Jiang P, Baranov E, Li S, et al. Tumor-targeting bacterial therapy with amino acid auxotrophs of GFP-expressing Salmonella typhimurium. Proc Natl Acad Sci U S A (2005) 102(3):755-60. doi:10.1073/ pnas.0408422102

12. Linnebacher M, Maletzki C, Klier U, Klar E. Bacterial immunotherapy of gastrointestinal tumors. Langenbecks Arch Surg (2012) 397(4):557-68. doi:10.1007/s00423-011-0892-6

13. Wood LM, Paterson Y. Attenuated Listeria monocytogenes: a powerful and versatile vector for the future of tumor immunotherapy. Front Cell Infect Microbiol (2014) 4:51. doi:10.3389/fcimb.2014.00051

\section{ETHICS STATEMENT}

Animal studies were carried out in accordance with, and after approval of the Animal Research Ethics Committee of the CMHS (Protocols \#A13/15 and A26/13).

\section{AUTHOR CONTRIBUTIONS}

SK performed experiments, analyzed data, and wrote the paper. AA-S contributed to the flowcytometric analysis. OC-M contributed in writing the paper. $\mathrm{MF}-\mathrm{C}$ wrote the final manuscript. BA- $R$ designed the study, supervised the project, analyzed data, and wrote the final manuscript.

\section{FUNDING}

This work was funded by grant \#NP-15-02 from the Research Grants Committee, College of Medicine \& Health Sciences and grant \#31M093 from the Office of Research and Sponsored Projects, UAE University (both to BA-R).

14. Wang Y, Toussaint B, Le Gouellec A. Bacterial vectors for the delivery of tumor antigens. Methods Mol Biol (2014) 1139:429-41. doi:10.1007/ 978-1-4939-0345-0_33

15. Balkwill FR, Capasso M, Hagemann T. The tumor microenvironment at a glance. J Cell Sci (2012) 125(Pt 23):5591-6. doi:10.1242/jcs.116392

16. Kerkar SP, Restifo NP. Cellular constituents of immune escape within the tumor microenvironment. Cancer Res (2012) 72(13):3125-30. doi:10.1158/ 0008-5472.CAN-11-4094

17. Galdiero MR, Bonavita E, Barajon I, Garlanda C, Mantovani A, Jaillon S. Tumor associated macrophages and neutrophils in cancer. Immunobiology (2013) 218(11):1402-10. doi:10.1016/j.imbio.2013.06.003

18. Mantovani A, Sica A. Macrophages, innate immunity and cancer: balance, tolerance, and diversity. Curr Opin Immunol (2010) 22(2):231-7. doi:10.1016/j. coi.2010.01.009

19. Rodriguez PC, Quiceno DG, Zabaleta J, Ortiz B, Zea AH, Piazuelo MB, et al. Arginase I production in the tumor microenvironment by mature myeloid cells inhibits T-cell receptor expression and antigen-specific T-cell responses. Cancer Res (2004) 64(16):5839-49. doi:10.1158/0008-5472.CAN-04-0465

20. Mantovani A, Sica A, Sozzani S, Allavena P, Vecchi A, Locati M. The chemokine system in diverse forms of macrophage activation and polarization. Trends Immunol (2004) 25(12):677-86. doi:10.1016/j.it.2004.09.015

21. Martinez FO, Sica A, Mantovani A, Locati M. Macrophage activation and polarization. Front Biosci (2008) 13:453-61. doi:10.2741/2692

22. Qian BZ, Pollard JW. Macrophage diversity enhances tumor progression and metastasis. Cell (2010) 141(1):39-51. doi:10.1016/j.cell.2010.03.014

23. Kaimala S, Mohamed YA, Nader N, Issac J, Elkord E, Chouaib S, et al. Salmonella-mediated tumor regression involves targeting of tumor myeloid suppressor cells causing a shift to M1-like phenotype and reduction in suppressive capacity. Cancer Immunol Immunother (2014) 63(6):587-99. doi:10.1007/s00262-014-1543-x

24. Hong EH, Chang SY, Lee BR, Pyun AR, Kim JW, Kweon MN, et al. Intratumoral injection of attenuated Salmonella vaccine can induce tumor microenvironmental shift from immune suppressive to immunogenic. Vaccine (2013) 31(10):1377-84. doi:10.1016/j.vaccine.2013.01.006

25. Lizotte PH, Baird JR, Stevens CA, Lauer P, Green WR, Brockstedt DG, et al. Attenuated Listeria monocytogenes reprograms M2-polarized tumor-associated macrophages in ovarian cancer leading to iNOS-mediated tumor cell lysis. Oncoimmunology (2014) 3:e28926. doi:10.4161/onci.28926

26. Kalupahana RS, Mastroeni P, Maskell D, Blacklaws BA. Activation of murine dendritic cells and macrophages induced by Salmonella enterica serovar Typhimurium. Immunology (2005) 115(4):462-72. doi:10.1111/j. 1365-2567.2005.02180.x 
27. Akira S, Takeda K. Toll-like receptor signalling. Nat Rev Immunol (2004) 4:499-511. doi:10.1038/nri1391

28. Vendrell A, Gravisaco MJ, Pasetti MF, Croci M, Colombo L, Rodríguez C, et al. A novel Salmonella Typhi-based immunotherapy promotes tumor killing via an antitumor Th1-type cellular immune response and neutrophil activation in a mouse model of breast cancer. Vaccine (2011) 29(4):728-36. doi:10.1016/j. vaccine.2010.11.017

29. Grille S, Moreno M, Bascuas T, Marqués JM, Muñoz N, Lens D, et al. Salmonella enterica serovar Typhimurium immunotherapy for B-cell lymphoma induces broad anti-tumour immunity with therapeutic effect. Immunology (2014) 143(3):428-37. doi:10.1111/imm.12320

30. Baird JR, Fox BA, Sanders KL, Lizotte PH, Cubillos-Ruiz JR, Scarlett UK, et al. Avirulent Toxoplasma gondii generates therapeutic antitumor immunity by reversing immunosuppression in the ovarian cancer microenvironment. Cancer Res (2013) 73(13):3842-51. doi:10.1158/0008-5472.CAN-12-1974

31. Sanders KL, Fox BA, Bzik DJ. Attenuated Toxoplasma gondii stimulates immunity to pancreatic cancer by manipulation of myeloid cell populations. CancerImmunolRes (2015) 3(8):891-901. doi:10.1158/2326-6066.CIR-14-0235

32. Bellora F, Castriconi R, Dondero A, Pessino A, Nencioni A, Liggieri G, et al. TLR activation of tumor-associated macrophages from ovarian cancer patients triggers cytolytic activity of NK cells. Eur J Immunol (2014) 44(6): 1814-22. doi:10.1002/eji.201344130

33. Jewett A, Tseng HC. Tumor induced inactivation of natural killer cell cytotoxic function; implication in growth, expansion and differentiation of cancer stem cells. J Cancer (2011) 2:443-57. doi:10.7150/jca.2.443

34. Zitvogel L, Tesniere A, Kroemer G. Cancer despite immunosurveillance: immunoselection and immunosubversion. Nat Rev Immunol (2006) 6(10):715-27. doi:10.1038/nri1936

35. Vitale M, Cantoni C, Pietra G, Mingari MC, Moretta L. Effect of tumor cells and tumor microenvironment on NK-cell function. Eur JImmunol (2014) 44(6):1582-92. doi:10.1002/eji.201344272

36. al-Ramadi BK, Mustafa N, AbouHaidar M, Fernandez-Cabezudo MJ. Induction of innate immunity by IL-2-expressing Salmonella confers protection against lethal infection. Mol Immunol (2003) 39(13):763-70. doi:10.1016/ S0161-5890(03)00005-1

37. al-Ramadi BK, Fernandez-Cabezudo MJ, El-Hasasna H, Al-Salam S, Bashir G, Chouaib S. Potent anti-tumor activity of systemically-administered IL2expressing Salmonella correlates with decreased angiogenesis and enhanced tumor apoptosis. Clin Immunol (2009) 130(1):89-97. doi:10.1016/j. clim.2008.08.021

38. al-Ramadi BK, Adeghate E, Mustafa N, Ponery AS, Fernandez-Cabezudo MJ. Cytokine expression by attenuated intracellular bacteria regulates the immune response to infection: the Salmonella model. Mol Immunol (2002) 38(12-13):931-40. doi:10.1016/S0161-5890(02)00020-2

39. al-Ramadi BK, Al-Dhaheri MH, Mustafa N, Abouhaidar M, Xu D, Liew FY, et al. Influence of vector-encoded cytokines on anti-Salmonella immunity: divergent effects of interleukin-2 and tumor necrosis factor alpha. Infect Immun (2001) 69(6):3980-8. doi:10.1128/IAI.69.6.3980-3988.2001

40. Fernandez-Cabezudo MJ, Mechkarska M, Azimullah S, al-Ramadi BK. Modulation of macrophage proinflammatory functions by cytokine-expressing Salmonella vectors. Clin Immunol (2009) 130(1):51-60. doi:10.1016/j.clim. 2008.08.017

41. Yoon W, Park YC, Kim J, Chae YS, Byeon JH, Min SH, et al. Application of genetically engineered Salmonella typhimurium for interferon-gammainduced therapy against melanoma. Eur J Cancer (2017) 70:48-61. doi:10.1016/j. ejca.2016.10.010

42. Bascuas T, Moreno M, Grille S, Chabalgoity JA. Salmonella immunotherapy improves the outcome of CHOP chemotherapy in non-Hodgkin lymphoma-bearing mice. Front Immunol (2018) 9:7. doi:10.3389/fimmu.2018.00007

43. Okada R, Kondo T, Matsuki F, Takata H, Takiguchi M. Phenotypic classification of human CD4+ T cell subsets and their differentiation. Int Immunol (2008) 20(9):1189-99. doi:10.1093/intimm/dxn075

44. Huang H, Hao S, Li F, Ye Z, Yang J, Xiang J. CD4+ Th1 cells promote CD8+ Tc1 cell survival, memory response, tumor localization and therapy by targeted delivery of interleukin 2 via acquired pMHC I complexes. Immunology (2007) 120(2):148-59. doi:10.1111/j.1365-2567.2006.02452.x

45. Knutson KL, Disis ML. Tumor antigen-specific Thelper cells in cancer immunity and immunotherapy. Cancer Immunol Immunother (2005) 54(8):721-8. doi:10.1007/s00262-004-0653-2
46. Chung AS, Wu X, Zhuang G, Ngu H, Kasman I, Zhang J, et al. An interleukin-17-mediated paracrine network promotes tumor resistance to antiangiogenic therapy. Nat Med (2013) 19(9):1114-23. doi:10.1038/nm.3291

47. Oelkrug C, Ramage JM. Enhancement of T cell recruitment and infiltration into tumours. Clin Exp Immunol (2014) 178(1):1-8. doi:10.1111/cei.12382

48. Vendrell A, Gravisaco MJ, Goin JC, Pasetti MF, Herschllik L, De Toro J, et al. Therapeutic effects of Salmonella typhi in a mouse model of T-cell lymphoma. J Immunother (2013) 36(3):171-80. doi:10.1097/CJI.0b013e3182886d95

49. Grille S, Moreno M, Brugnini A, Lens D, Chabalgoity JA. A therapeutic vaccine using Salmonella-modified tumor cells combined with interleukin-2 induces enhanced antitumor immunity in B-cell lymphoma. Leuk Res (2013) 37(3):341-8. doi:10.1016/j.leukres.2012.10.003

50. Lee CH, Hsieh JL, Wu CL, Hsu PY, Shiau AL. T cell augments the antitumor activity of tumor-targeting Salmonella. Appl Microbiol Biotechnol (2011) 90(4):1381-8. doi:10.1007/s00253-011-3180-Z

51. Zhao M, Yang M, Ma H, Li X, Tan X, Li S, et al. Targeted therapy with a Salmonella typhimurium leucine-arginine auxotroph cures orthotopic human breast tumors in nude mice. Cancer Res (2006) 66(15):7647-52. doi:10.1158/0008-5472.CAN-06-0716

52. Murakami T, Hiroshima Y, Zhang Y, Zhao M, Kiyuna T, Hwang HK, et al. Tumor-targeting Salmonella typhimurium A1-R promotes tumoricidal CD8(+) T cell tumor infiltration and arrests growth and metastasis in a syngeneic pancreatic-cancer orthotopic mouse model. J Cell Biochem (2018) 119(1):634-9. doi:10.1002/jcb.26224

53. Kim SH, Castro F, Paterson Y, Gravekamp C. High efficacy of a Listeria-based vaccine against metastatic breast cancer reveals a dual mode of action. Cancer Res (2009) 69(14):5860-6. doi:10.1158/0008-5472.CAN-08-4855

54. Olino K, Wada S, Edil BH, Pan X, Meckel K, Weber W, et al. Tumor-associated antigen expressing Listeria monocytogenes induces effective primary and memory T-cell responses against hepatic colorectal cancer metastases. Ann Surg Oncol (2012) 19(Suppl 3):S597-607. doi:10.1245/s10434-011-2037-0

55. Gogoi D, Chiplunkar SV. Targeting gamma delta T cells for cancer immunotherapy: bench to bedside. Indian J Med Res (2013) 138(5):755-61.

56. Fowler DW, Copier J, Wilson N, Dalgleish AG, Bodman-Smith MD. Mycobacteria activate gammadelta T-cell anti-tumour responses via cytokines from type 1 myeloid dendritic cells: a mechanism of action for cancer immunotherapy. Cancer Immunol Immunother (2012) 61(4):535-47. doi:10.1007/ s00262-011-1121-4

57. Guirnalda PD, Paterson Y. Vaccination with immunotherapeutic Listeria monocytogenes induces IL-17(+) gammadelta T cells in a murine model for HPV associated cancer. Oncoimmunology (2012) 1(6):822-8. doi:10.4161/ onci.20491

58. Katoh H, Watanabe M. Myeloid-derived suppressor cells and therapeutic strategies in cancer. Mediators Inflamm (2015) 2015:159269. doi:10.1155/2015/159269

59. Lu T, Ramakrishnan R, Altiok S, Youn JI, Cheng P, Celis E, et al. Tumorinfiltrating myeloid cells induce tumor cell resistance to cytotoxic $\mathrm{T}$ cells in mice. J Clin Invest (2011) 121(10):4015-29. doi:10.1172/JCI45862

60. Wallecha A, Singh R, Malinina I. Listeria monocytogenes (Lm)-LLO immunotherapies reduce the immunosuppressive activity of myeloid-derived suppressor cells and regulatory $\mathrm{T}$ cells in the tumor microenvironment. J Immunother (2013) 36(9):468-76. doi:10.1097/CJI.00000000000000000

61. Maynard CL, Weaver CT. Diversity in the contribution of interleukin-10 to T-cell-mediated immune regulation. Immunol Rev (2008) 226:219-33. doi:10.1111/j.1600-065X.2008.00711.X

62. Chandra D, Jahangir A, Quispe-Tintaya W, Einstein MH, Gravekamp C. Myeloid-derived suppressor cells have a central role in attenuated Listeria monocytogenes-based immunotherapy against metastatic breast cancer in young and old mice. Br J Cancer (2013) 108(11):2281-90. doi:10.1038/ bjc.2013.206

63. Zhang Y, Luo F, Li A, Qian J, Yao Z, Feng X, et al. Systemic injection of TLR1/2 agonist improves adoptive antigen-specific $\mathrm{T}$ cell therapy in glioma-bearing mice. Clin Immunol (2014) 154(1):26-36. doi:10.1016/j.clim.2014.06.004

64. Josefowicz SZ, Lu LF, Rudensky AY. Regulatory T cells: mechanisms of differentiation and function. Annu Rev Immunol (2012) 30:531-64. doi:10.1146/ annurev.immunol.25.022106.141623

65. Liu T, Chopra AK. An enteric pathogen Salmonella enterica serovar Typhimurium suppresses tumor growth by downregulating CD44high and $\mathrm{CD} 4 \mathrm{~T}$ regulatory (Treg) cell expression in mice: the critical role of 
lipopolysaccharide and Braun lipoprotein in modulating tumor growth. Cancer Gene Ther (2010) 17(2):97-108. doi:10.1038/cgt.2009.58

66. Zhang Y, Luo F, Cai Y, Liu N, Wang L, Xu D, et al. TLR1/TLR2 agonist induces tumor regression by reciprocal modulation of effector and regulatory $\mathrm{T}$ cells. J Immunol (2011) 186(4):1963-9. doi:10.4049/jimmunol.1002320

67. Felgner S, Kocijancic D, Frahm M, Weiss S. Bacteria in cancer therapy: renaissance of an old concept. Int JMicrobiol (2016) 2016:8451728. doi:10.1155/2016/8451728

68. Kocijancic D, Felgner S, Schauer T, Frahm M, Heise U, Zimmermann K, et al. Local application of bacteria improves safety of Salmonella-mediated tumor therapy and retains advantages of systemic infection. Oncotarget (2017) 8(30):49988-50001. doi:10.18632/oncotarget.18392

69. Heap JT, Theys J, Ehsaan M, Kubiak AM, Dubois L, Paesmans K, et al. Spores of Clostridium engineered for clinical efficacy and safety cause regression and cure of tumors in vivo. Oncotarget (2014) 5(7):1761-9. doi:10.18632/ oncotarget.1761

70. Paterson Y, Guirnalda PD, Wood LM. Listeria and Salmonella bacterial vectors of tumor-associated antigens for cancer immunotherapy. Semin Immunol (2010) 22(3):183-9. doi:10.1016/j.smim.2010.02.002

71. Maciag PC, Radulovic S, Rothman J. The first clinical use of a live-attenuated Listeria monocytogenes vaccine: a phase I safety study of Lm-LLO-E7 in patients with advanced carcinoma of the cervix. Vaccine (2009) 27(30):3975-83. doi:10.1016/j.vaccine.2009.04.041

72. Le DT, Brockstedt DG, Nir-Paz R, Hampl J, Mathur S, Nemunaitis J, et al. A live-attenuated Listeria vaccine (ANZ-100) and a live-attenuated Listeria vaccine expressing mesothelin (CRS-207) for advanced cancers: phase I studies of safety and immune induction. Clin Cancer Res (2012) 18(3):858-68. doi:10.1158/1078-0432.CCR-11-2121

73. Felgner S, Frahm M, Kocijancic D, Rohde M, Eckweiler D, Bielecka A, et al. aroA-deficient Salmonella enterica serovar Typhimurium is more than a metabolically attenuated mutant. MBio (2016) 7(5):e01220-16. doi:10.1128/ mBio.01220-16

74. Felgner S, et al. Engineered Salmonella enterica serovar Typhimurium overcomes limitations of anti-bacterial immunity in bacteria-mediated tumor therapy Oncoimmunology (2018) 7(2):e1382791. doi:10.1080/2162402X.2017.1382791

75. Frahm M, Felgner S, Kocijancic D, Rohde M, Hensel M, Curtiss R III, et al. Efficiency of conditionally attenuated Salmonella enterica serovar Typhimurium in bacterium-mediated tumor therapy. MBio (2015) 6(2):e00254-15. doi:10.1128/mBio.00254-15

76. al-Ramadi BK, Brodkin MA, Mosser DM, Eisenstein TK. Immunosuppression induced by attenuated Salmonella. Evidence for mediation by macrophage precursors. J Immunol (1991) 146(8):2737-46.

77. al-Ramadi BK, Meissler JJ Jr, Huang D, Eisenstein TK. Immunosuppression induced by nitric oxide and its inhibition by interleukin-4. Eur J Immunol (1992) 22(9):2249-54. doi:10.1002/eji.1830220911

78. Underwood JC. Lymphoreticular infiltration in human tumours: prognostic and biological implications: a review. Br J Cancer (1974) 30(6):538-48. doi:10.1038/bjc.1974.233

Conflict of Interest Statement: The authors declare that the research was conducted in the absence of any commercial or financial relationships that could be construed as a potential conflict of interest.

Copyright (๑ 2018 Kaimala, Al-Sbiei, Cabral-Marques, Fernandez-Cabezudo and Al-Ramadi. This is an open-access article distributed under the terms of the Creative Commons Attribution License (CC BY). The use, distribution or reproduction in other forums is permitted, provided the original author(s) and the copyright owner are credited and that the original publication in this journal is cited, in accordance with accepted academic practice. No use, distribution or reproduction is permitted which does not comply with these terms. 